\title{
Formation and evolution of hybrid He-CO white dwarfs and their properties
}

\author{
Yossef Zenati ${ }^{1}$, Silvia Toonen ${ }^{1,2}$ and Hagai B. Perets ${ }^{1}$ \\ ${ }^{1}$ Physics Department, Technion - Israel Institute of Technology, Haifa 3200004, Israel \\ 2 Anton Pannekoek Institute for Astronomy, University of Amsterdam, 1090 GE, Amsterdam, The Netherlands
}

Accepted XXX. Received YYY; in original form ZZZ

\begin{abstract}
White dwarfs (WDs) are the stellar core remnants of low mass $\left(\lesssim 8 \mathrm{M}_{\odot}\right)$ stars. They are typically divided into three main composition groups: Oxygen-Neon (ONe), CarbonOxygen (CO) and Helium (He) WDs. The evolution of binary systems can significantly change the evolution of the binary stellar components. In particular, striping the envelope of an evolved star can give rise to a core remnant, which can later evolve into a WD with significantly different composition. Here we focus on the formation and evolution of hybrid HeCO WDs. We follow the formation and stellar evolution of such WDs for a range of initial conditions and provide their detailed structure, mass-radius relation and luminousity-temperature evolution. We find that both lowmass WDs $\left(<0.45 \mathrm{M}_{\odot}\right.$, typically thought to be He-WDs) and intermediate-mass WDs $\left(0.45<\mathrm{M}_{\mathrm{WD}} \leq 0.7\right.$, typically thought to be CO-WDs) could in fact be hybrid $\mathrm{HeCO}$ WDs, with $5-25(75-95) \%$ of their mass in $\mathrm{He}(\mathrm{CO})$. We use population synthesis calculations to infer the birth rate and properties of such WDs. We find that hybrid HeCO-WD comprise the majority of young $(<2 \mathrm{Gyr})$ WDs in binaries, but are more rare among older WDs in binaries. The high frequency and large He content of such WDs could have an important role in WD-WD mergers, and may give rise to sub-Chandrasekhar thermonuclear supernova explosions.
\end{abstract}

Key words: (stars:) white dwarfs - stars: evolution - stars: mass-loss - stars: horizontal branch

\section{INTRODUCTION}

White dwarfs (WDs) are the stellar core remnants of low mass $\left(\lesssim 8 \mathrm{M}_{\odot}\right)$ stars that formed following their post mainsequence (MS) evolution. They are composed mostly of electron-degenerate matter, and are divided into several types, including WDs composed of carbon-oxygen (CO) and Oxygen-Neon (ONe) WDs, corresponding to the stellar evolutionary end points of intermediate and high mass stars, respectively. The minimum mass of a present day WD formed through stellar evolution of a single star is in the range $\sim 0.50-0.52 \mathrm{M}_{\odot}$ arising from the lowest mass stars with lifetimes shorter than the age of the universe (1.01 down to $0.84 \mathrm{M}_{\odot}$ for $\mathrm{Z}=1.5 \mathrm{Z}_{\odot}$ down-to $\mathrm{Z}=0.1 \mathrm{Z}_{\odot}$, respectively, as calculated from stellar evolution models using the SeBa module (Toonen et al. 2012). Such WDs would be CO WDs. However, binary evolution can change these outcomes and produce different types of WDs and allowing for a much lower mass range (e.g. Han et al. 2002; Rebassa-Mansergas et al. 2011, and references therein).

In interacting binaries each of the stellar component may fill its Roche lobe, and may be stripped of part of its hydrogen/helium-rich envelope during its evolution on the red giant branch (RGB; or the asymptotic giant branch, AGB) stage. Such altered evolution can give rise to qualitatively different evolution and the formation of present day very low mass $\left(\mathrm{VLM} ;<0.45 \mathrm{M}_{\odot}\right.$; e.g. Han et al. 2002) WDs. The evolution and final outcomes of the binary evolution strongly depend on the initial conditions: the mass of the stellar components and their initial separation. In particular, WDs of masses lower than $0.45 \mathrm{M}_{\odot}$ are typically thought to be Helium (He)-WDs formed through this channel (e.g. Iben \& Tutukov 1985; Han et al. 2002; Nelemans et al. 2001; Istrate et al. 2016; Zhang et al. 2018). However, the complex binary evolution channel can give rise to VLM (as well as more massive) hybrid-WDs, composed of significant fractions of both $\mathrm{CO}$ and He. Such white dwarfs descend from stars which fill their Roche lobes in the stage of hydrogen burning in a shell, become hot sub-dwarfs in the He-burning stage, but do not experience envelope expansion after the formation of a degenerate carbon-oxygen core (Iben \& Tutukov 1985; Nelemans et al. 2000).

The exact definition of a hybrid HeCO WD is somewhat arbitrary; one can consider a hybrid HeCO WD as any WD in which no less than some fraction $f$ of the mass is com- 
posed of $\mathrm{He}$, and no less of a fraction $f$ of the mass is composed of CO. In practice, in all of the hybrid WDs we find the the mass is dominated by CO. Here we focus on such hybrid WDs; we explore their properties and evolutionary channels, and discuss their implications on our understanding of WDs and their structure, which are strongly dependent on the composition. Not less important, HeCO WDs may have an important role in affecting the outcomes of WD-mergers, and in particular the possible production of thermonuclear $\mathrm{SNe}$ from double-degenerate WD mergers. For the latter, the existence of a significant mass in He can catalyze thermonuclear SN explosions even in sub-Chandrasekhar mass WDs (e.g. Woosley et al. 1986; Iben et al. 1987; Livne \& Glasner 1990; Bildsten et al. 2007; Waldman et al. 2011). Hence, understanding of hybrid WDs is critical for a wide range of compact objects as well as their potential explosive mergers.

HeCO WDs have been first discussed by Iben \& Tutukov (1985) who suggested that a significant fraction of the VLM WDs could be HeCO WDs rather than $\mathrm{He}$ (only) WDs. The possibility of HeCO WDs, later termed hybrid WDs, have been then further discussed (though briefly) in various contexts (Tutukov \& Yungelson 1992; Iben et al. 1997; Nelemans et al. 2000). Studies by Althaus et al. (2004) and Panei et al. (2007) explored the evolution of VLMs from low mass $\left(<3.16 \mathrm{M}_{\odot}\right)$ progenitor stars, and showed that Oxygen-core VLMs can be formed through binary evolution, producing WDs in the mass range $0.35-0.45 \mathrm{M}_{\odot}$ mostly composed of Oxygen. Follow-up studies by Prada Moroni \& Straniero (2009) had been able to produce a CO WD of $0.33 \mathrm{M}_{\odot}$ suggesting it as the lower limit for such WDs. More recent studies have further developed the study of VLM He WDs (Istrate et al. 2016; Zhang et al. 2018, and references therein). All of these later studies explored the possibility of VLM He WDs, with little discussion of the potential of hybrid He-CO VLMs. Our focus is exploring the range of possible hybrid $\mathrm{HeCO}$ WDs and their properties, map the possible range of He to CO mass fractions in these WDs and characterize their structure and mass-radius relationship. Our detailed stellar evolution findings (using the MESA code; Paxton et al. 2011, 2015) can be used to direct the more simplified population synthesis models in order to characterize the general properties of hybrid-WD that form, their binary system progenitors and the type of double-degenerate mergers in which they participate. The outcomes of the latter are further explored in forthcoming publications.

The paper is structured as follows. First (section 2), we describe the methods used to explore the detailed stellar evolution models (using the MESA code) and population studies (using the SeBa population synthesis code; section 3) for the formation of hybrid-WDs. We present our main results in section 4 and then discuss the implications of hybrid-WDs, and summarize (section 5).

\section{DETAILED STELLAR EVOLUTION: METHODS}

Hybrid HeCO WDs can evolve from intermediate mass stars in binary systems through through a phase of mass transfer through Roch-lobe overflow (RLOF) or shared common- envelope evolution (see e.g. Ivanova et al. 2013). In the interacting binary the companion envelope can be stripped following the formation of a He core and the evolution on the red giant branch (RGB). The later evolution of the stripped star and the He core is then significantly altered compared with the uninterrupted evolution of a non-interacting (single) star. When most of the red giant envelope is removed, hydrogen shell burning is quelled, the He-core keeps growing, and the star begins to contract. If the He core is sufficiently massive, the contraction will eventually trigger $\mathrm{He}$ ignition (see Iben \& Tutukov 1985) and the formation of a $\mathrm{CO}$ core; the He to $\mathrm{CO}$ ratio will then be determined by the specific detailed evolution of He burning into $\mathrm{CO}$, and mass-loss through winds from the envelope.

In order to follow the complex evolution, we begin by considering a range of initial binary conditions. The initial binary separations are chosen such that the lower mass binary component will eventually fill its Roche lobe. The stellar evolutionary tracks of binary components are followed from the pre-MS stage until the final production of the WD. We mapped these conditions into the stellar evolution code MESA and the used the binary mode (Paxton et al. 2015) to follow their evolution for the range of initial conditions. We also tested models where the binary evolution (leading to effective mass-loss) had been introduced by us into single star models. Mass loss was introduced in these cases when the conditions for the radius and core mass are such that RLOF is expected. In practice, this point is not fine-tuned, and these conditions can be fulfilled over a range of stellar radii (as indicated in Table 1) once the star experiences the first thermal pulse, or in some cases the second or third thermal pulses. In this range the final outcomes of the evolution produce qualitatively and quantitatively similar results throughout our simulations, we therefore show the results only for the choice of the middle point in the relevant range).

In all cases where both the binary and single mode models converged we obtained consistent final outcomes, further validating both these approaches. In some cases, however, only our altered single mode model numerically converged, in which case we report the results only from the single-mode model. The properties of the initial binaries extend over a range of initial primary component mass $\left(2.5 \mathrm{M}_{\odot} \leq \mathrm{M}_{\text {donor }} \leq 4 \mathrm{M}_{\odot}\right)$ and mass ratios $\left(0.65 \lesssim \mathrm{q}=\mathrm{M}_{\text {donor }} / \mathrm{M}_{\text {companion }} \lesssim 0.81\right.$; with the exception of one case with $q=0.36$ ); the detailed models and their outcomes are summarized in Table 1 . We also modeled donor stars with lower masses $\left(<2 \mathrm{M}_{\odot}\right)$, but these only produced $\mathrm{He}$ WDs, or He WDs with very low fraction of CO; also consistent with results by Prada Moroni \& Straniero (2009).

Though some models had been difficult to converge numerically, we were able to resolve the evolution of these binaries through the whole relevant mass range and mass ratio. In all these cases we were able to produce a hybrid $\mathrm{HeCO}$ WD. In the following we discuss in detail our assumptions and initial conditions. All of our models (and MESA inlist files) are openly available at GitHub as to enable simple reproduction of the results (github.com/Hybrid-WD).

In the following we list our detailed assumptions and considerations.

- Metallicity: All models are of of Solar metallicity stars $\mathrm{Z}=\mathrm{Z}_{\odot}=0.02$. 
- Mass transfer: we use a range of $10^{-8}-10^{-10} \mathrm{M}_{\odot} \mathrm{yr}^{-1}$ for the min mdot for implicit parameter.

- Mixing: We set $\alpha=1 / \mathrm{H}_{\mathrm{p}}=1.5$ as the ratio of typical mixing length to the local pressure scale height, where $l$ is the mixing length, $\alpha$ is a free parameter, and $\mathrm{H}_{\mathrm{p}}$ is the pressure scale height. Semi-convective mixing and thermohaline mixing are taken to be 0.01 and 2, respectively (Farmer et al. 2016).

- Nuclear reaction network: We use a 75 isotope nuclear reaction network, containing the relevant isotopes needed for He, carbon and oxygen burning.

- Stopping condition: We stop the evolution once the star becomes fully degenerate WD and no further evolution, beside WD cooling is observed. Our condition effectively translates to WD luminosity and temperature that fall below $\mathrm{L} \leq 1.8 \mathrm{~L}_{\odot} ; \mathrm{T}_{\text {eff }} \leq 5.1 \mathrm{~T}_{\odot, \text { eff }}$ (or in some cases, $\left.\mathrm{L} \leq 1.12 \mathrm{~L}_{\odot} ; \mathrm{T}_{\text {eff }} \leq 4.94 \mathrm{~T}_{\odot, \text { eff }}\right)$, respectively.

- Mass loss: The effective mass loss is introduced through the use of Reimers formulation $\mathrm{M}=-4 \times 10^{-13} \eta_{\mathrm{R}} \cdot \mathrm{L} \cdot \mathrm{R} \cdot \mathrm{M} / \mathrm{L}_{\odot} \mathrm{R}_{\odot} \mathrm{M}_{\odot}$ where $\eta$ is in the range $0.7 \lesssim \eta \lesssim 1$. In order to ensure that the mass of the star is appropriately adjusted following the mass loss, we let the star relax afterwards.

- Overshoot: The optimal overshoot parameter enabling numerical convergence for the whole mass range (for $\left.2.5-4 \mathrm{M}_{\odot}\right)$ was found to be $\mathrm{f}_{\mathrm{ov}}=0.0016$.

\section{POPULATION SYNTHESIS MODELS}

The formation and evolution of interacting binaries producing hybrid-WDs is simulated with the binary population synthesis (BPS) code SeBa (Portegies Zwart \& Verbunt 1996; Toonen et al. 2012; Toonen \& Nelemans 2013). SeBa is a fast code for simulating binary evolution based on parametrized stellar evolution, including processes such as mass transfer episodes, common-envelope evolution and stellar winds. We employ SeBa to generate a large population of binaries on the zero-age MS, we simulate their subsequent evolution, and extract those that produce hybrid - HeCO WDs.

It was shown in Toonen et al. (2014) that the main sources of differences between different BPS codes is due to the choice of input physics and initial conditions. Here we focus only on a specific set of choices, and use the detailed stellar evolution models to test the BPS models predictions for hybrid WDs. A wider range of initial conditions and physical assumptions will be explored in future work, where hybrid WDs may play a roles in the evolution of double degenerate (WD-WD, NS-WD and BH-WD) mergers.

The basic conditions and assumptions we use embody a classical set-up for BPS calculations, and the binaries are generated and evolved in the following way:

- The primary masses are drawn from a Kroupa IMF (Kroupa et al. 1993) with masses in the range between $0.1-100 \mathrm{M}_{\odot}$.

- The secondary masses are drawn from a uniform mass ratio distribution with $0<q \equiv M_{2} / M_{1}<1$ (Raghavan et al. 2010; Duchêne \& Kraus 2013; De Rosa et al. 2014).

- The orbital separations $a$ follow a uniform distribution in $\log (a)$ (Abt 1983)
- The initial eccentricities $e$ follow a thermal distribution (Heggie 1975).

- A binary fraction $\mathcal{B}$ of $50 \%$ which is appropriate for A-type primaries (Raghavan et al. 2010; Duchêne \& Kraus 2013).

- Hybrid definition: Unless stated otherwise, in the BPS calculations a hybrid is defined as a WD with at least $5 \%$ of its mass composed of He (see Eq. 1).

- We allow bare degenerate helium cores to ignite if the core gets exposed (through binary interactions) when its mass is within $0.02 \mathrm{M}_{\odot}$ of the mass where the helium flash happens (Han et al. 2002; Nelemans 2010).

- We construct two BPS models that differ with respect to the common-envelope phase. This is a short phase in the evolution of a binary system when both stars share a common-envelope. Despite its strong effect on the binary orbit, common-envelope evolution is poorly understood (see e.g. Ivanova et al. 2013, for a review). We replicate model $\alpha \alpha$ and model $\gamma \alpha$ from Toonen et al. (2017), where the prior is based on the classical energy balance during the commonenvelope phase, whereas the latter is based on a balance of angular momentum.

In principle, hybrid HeCO WDs can be formed in two ways, either through the common envelope or the RLOF channels. In the interacting binary the hydrogen-rich envelope of the hybrid progenitor can be stripped following the formation of a He core and the evolution on the RGB or AGB. The later evolution of the stripped star and the He core is then significantly altered compared with the uninterrupted evolution of a non-interacting (single) star. When most of the giant envelope is removed, hydrogen shell burning is quelled, giving rise to a helium star, that can be observed as a possible $\mathrm{SdB}$ star. If the He core is sufficiently massive $\left(\mathrm{M}_{c}>0.32 \mathrm{M}_{\odot}\right)$, He ignition can be triggered (see Iben \& Tutukov 1985, leading to the formation of a $\mathrm{CO}$ core; the He to $\mathrm{CO}$ ratio will then be determined by the specific detailed evolution. For the intermediate mass stars in this channel, the He ignites under non-degenerate conditions).

In the second evolutionary channel for lower-mass stars (typically $<2 \mathrm{M}_{\odot}$ ), ignition of the He occurs under degenerate conditions. In this channel the progenitors are stripped close to the peak of first giant branch, contract, and then ignite (see Han et al. 2002, for details). However, Our detailed stellar evolution models show that WDs produced through this channel are effectively He WDs which contain only a very small fraction of $\mathrm{CO}(<1 \%)$. Nevertheless, foe completeness we consider the BPS of such WDs, but divide the contributions between the main channel and this latter channel, which does not produce bona-fide hybrid HeCO WDs. As discussed below the contribution from the low-mass progenitors is small and does not significantly affect any of our conclusions regarding hybrid WDs.

\section{RESULTS}

\subsection{Detailed stellar evolution models}

Table 1 and figures 1-5 summarize the results for the mass, composition and structure of hybrid WDs as a function of their final mass, resulting from our detailed stellar evolution models. Although hybrids of different masses are produced 


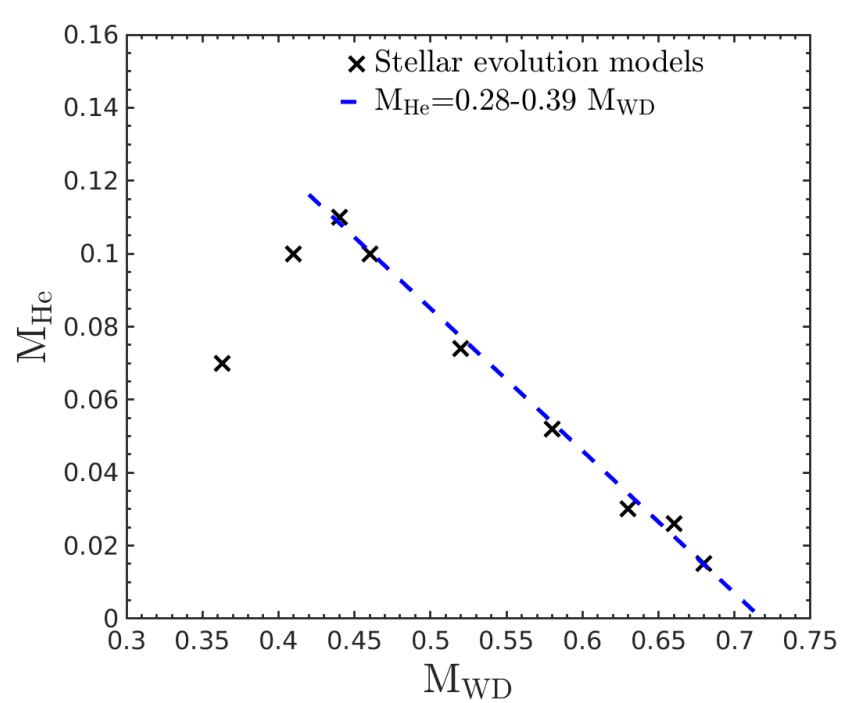

Figure 1. The He mass vs. total mass of He WDs from the detailed stellar evolution models. The blue line depicts a linear fit for the range $0.4 \leq \mathrm{M}_{\mathrm{WD}} \leq 0.72$.

from binaries which differ in various aspects (initial mass, mass ratio, separation etc.), the final compositions form a fairly continuous sequence for most of the mass range, with He mass fractions in the range $2-25 \%$ (see Fig. 1). As expected, the He forms an outer shell around the CO core, with only a very small layer of mixed CO-He composition (see Fig. 5).

Our detailed stellar evolution models suggest that the He mass in the WD $M_{\mathrm{He}}$ can be approximated by:

$\mathrm{M}_{\mathrm{He}}=0.28-0.39 \mathrm{M}_{\mathrm{WD}}$,

for most of the mass range $\left(0.4 \leq \mathrm{M}_{\mathrm{WD}} \leq 0.7\right.$, where $M_{\mathrm{WD}}$ is the mass of the WD. This is also approximately consistent with estimates for the core mass of He stars at which point He burning is quenched as found by Hurley et al. (2000, see their Eq. 89) and implies there is a maximum mass for hybrids. The maximum mass of a hybrid WD is therefore $\sim 0.72 \mathrm{M}_{\odot}$, for which the He mass goes to zero. However, for a more conservative limit, requiring a significant fraction of the WD mass to be in $\mathrm{He}$ (e.g. > 5\%), the maximum mass becomes $0.63 \mathrm{M}_{\odot}$. A lower limit to the mass of a hybrid is expected to be around $\sim 0.32 \mathrm{M}_{\odot}$, as below the core is not massive enough to ignite He burning (e.g. Han et al. 2002).

\subsubsection{Evolution on the HR diagram}

In Fig. 2 we show the evolution of the HeCO WD progenitor on the HR diagram for six of the models in Table 1 covering the full mass range (the other three are not shown for clarity, but they behave very similarly). As can be seen the evolution can be quite complex, and though we are mostly interested in the final WD configuration we refer the interested reader to Iben \& Tutukov (1985) for a detailed discussion of the various evolutionary stages before the final formation of the hybrid WD. In Fig. 3 we compare the results from the MESA binary evolution model to a more simplified single star evolution where the envelope stripping is included artificially without fully following the binary evolution. As can
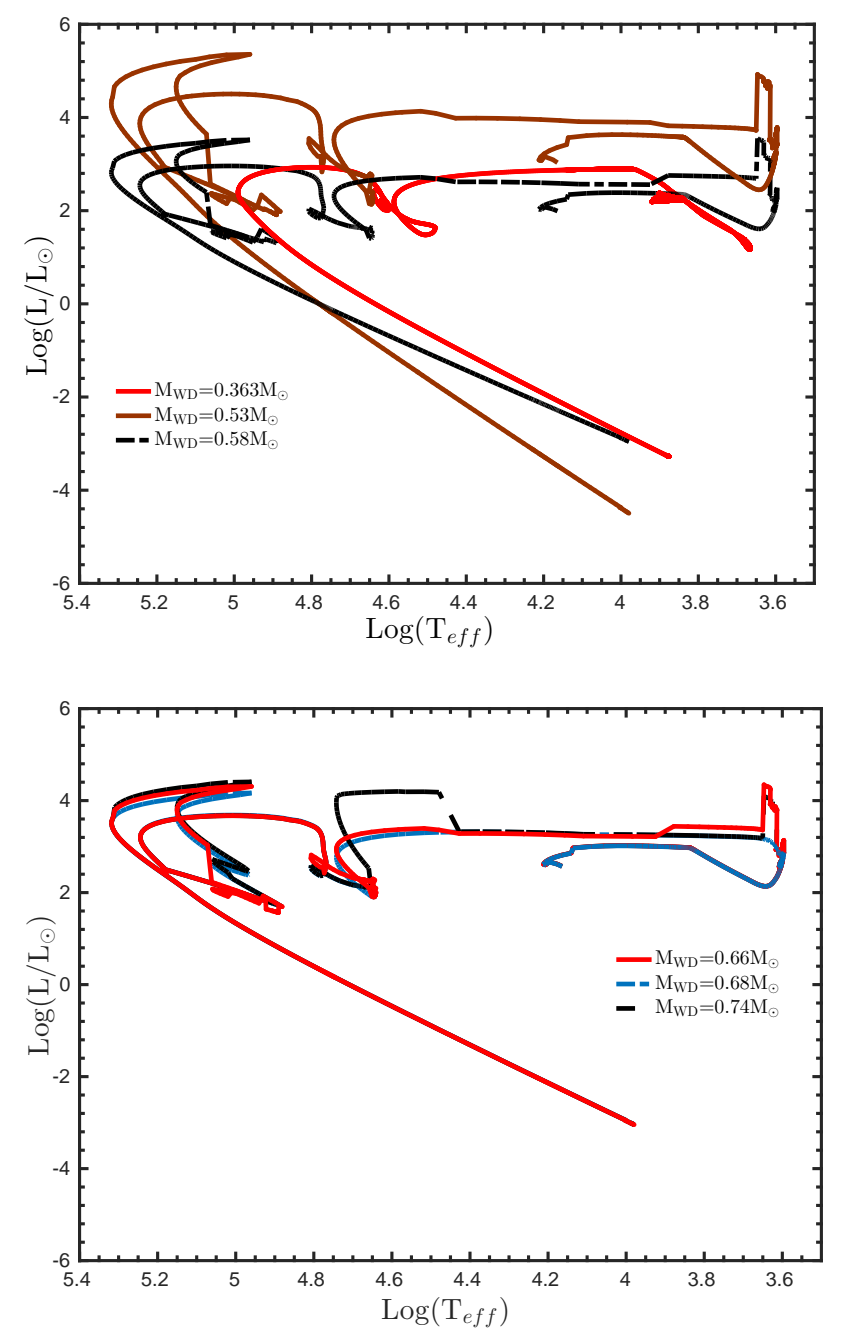

Figure 2. The HR diagram for the evolution of binaries producing hybrid HeCO WDs.

be seen the single models follow a different evolution at early times, but following the stripping they results in the same WD configuration and evolution, supporting their general use for modeling hybrids. This also suggests that uncertainties in the mass-transfer phase do not significantly affect the final structure of the hybrid WDs. Overall we find only small differences in the final WD configurations when we use the single and binary models, with typical C, O and He masses differences of less than $1 \%$ (besides the case of $\mathrm{M}_{\mathrm{WD}}=0.58$ where a $5 \%$ difference in the He abundance was found).

\subsection{Structure and composition of of HeCO hybrid WDs}

The mass-radius relation for the HeCO WDs is shown in Fig. 4. As expected the HeCO WDs radii typically fall in between those of CO WDs and purely He WDs.

\subsection{Population synthesis results}

We find that hybrid WDs are commonly formed. For a group of stars with a combined initial mass of $1000 \mathrm{M}_{\odot}$, we expect 


\begin{tabular}{ccccccccccc}
\hline$\#$ & $\mathrm{M}_{1}\left[\mathrm{M}_{\odot}\right]$ & $\mathrm{q}$ & $\mathrm{P}[\mathrm{day}]$ & $\log _{\text {eff }}$ & $\mathrm{M}_{\mathrm{WD}}^{\mathrm{f}}\left[\mathrm{M}_{\odot}\right]$ & $\mathrm{M}_{\mathrm{He} 4}^{\mathrm{Mesa}}\left[\mathrm{M}_{\odot}\right]$ & $\mathrm{M}_{\mathrm{Cl} 2}^{\mathrm{Mesa}}\left[\mathrm{M}_{\odot}\right]$ & $\mathrm{M}_{\mathrm{O}}^{\mathrm{Mesa}}\left[\mathrm{M}_{\odot}\right]$ & $\mathrm{M}_{\mathrm{H} 1}^{\mathrm{Mesa}}\left[\mathrm{M}_{\odot}\right]$ & Modeling* $^{*}$ \\
\hline \hline 1 & 2.50 & 0.81 & 5.01 & 5.0 & 0.36 & $(19 \%),(0.07)$ & $(42 \%),(0.15)$ & $(39 \%),(0.14)$ & $5.4 \times 10^{-4}$ & $\mathrm{~S}, \mathrm{~B}$ \\
\hline 2 & 2.66 & 0.78 & 4.5 & 5.0 & 0.41 & $(25 \%),(0.1)$ & $(38 \%),(0.16)$ & $(37 \%),(0.15)$ & $5.0 \times 10^{-4}$ & $\mathrm{~B}$ \\
\hline 3 & 2.75 & 0.79 & 5.00 & 5.0 & 0.44 & $(25 \%),(0.11)$ & $(37 \%),(0.16)$ & $(38 \%),(0.17)$ & $6 \times 10^{-4}$ & $\mathrm{~S}$ \\
\hline 4 & 2.85 & 0.36 & 5.25 & 5.2 & 0.46 & $(21 \%),(0.10)$ & $(38 \%),(0.17)$ & $(41 \%),(0.19)$ & $3 \times 10^{-4}$ & $\mathrm{~B}$ \\
\hline 5 & 3.01 & 0.74 & 6.28 & 5.0 & 0.53 & $(14 \%),(0.074)$ & $(43 \%),(0.23)$ & $(43 \%),(0.23)$ & $2 \times 10^{-5}$ & $\mathrm{~S}, \mathrm{~B}$ \\
\hline 6 & 3.20 & 0.71 & 6.28 & 5.2 & 0.58 & $(9 \%),(0.052)$ & $(45 \%),(0.26)$ & $(46 \%),(0.26)$ & $4.1 \times 10^{-5}$ & $\mathrm{~S}, \mathrm{~B}$ \\
\hline 8 & 3.41 & 0.70 & 7.00 & 5.2 & 0.63 & $(5 \%),(0.03)$ & $(48 \%),(0.3)$ & $(47 \%),(0.29)$ & $3.0 \times 10^{-4}$ & $\mathrm{~S}$ \\
\hline 9 & 3.50 & 0.77 & 7.44 & 5.2 & 0.66 & $(4 \%),(0.026)$ & $(48 \%),(0.31)$ & $(48 \%),(0.31)$ & $3.2 \times 10^{-4}$ & $\mathrm{~S}, \mathrm{~B}$ \\
\hline 10 & 4.00 & 0.78 & 11.13 & 5.2 & 0.74 & $(<1.5 \%),(<0.01)$ & $(49 \%),(0.36)$ & $(49 \%),(0.36)$ & $7.3 \times 10^{-4}$ & $\mathrm{~S}, \mathrm{~B}$ \\
\hline
\end{tabular}

*Type of modeling using the binary (B) evolution mode in MESA or an effective binary evolution using a single (S) star module.

Table 1. Characteristics of a binary succeed to survive until the hybrid WD phase. The $\mathrm{M}_{1}$ is the donor mass, $\mathrm{q}$ is the mass ratio ,P is the initial period for the binary in RLOF. $\log \mathrm{T}_{\text {eff }}$ is the effective temperature when the donor get our condition. $\mathrm{M}_{\mathrm{WD}}^{\mathrm{f}}$ is the final mass of the donor (hybrid WD mass), $\mathrm{M}_{\mathrm{He} 4}^{\mathrm{f}}, \mathrm{M}_{\mathrm{C} 12}^{\mathrm{f}}, \mathrm{M}_{\mathrm{O} 16}^{\mathrm{f}}$ is the final amount of He4, C12, $\mathrm{O} 16$

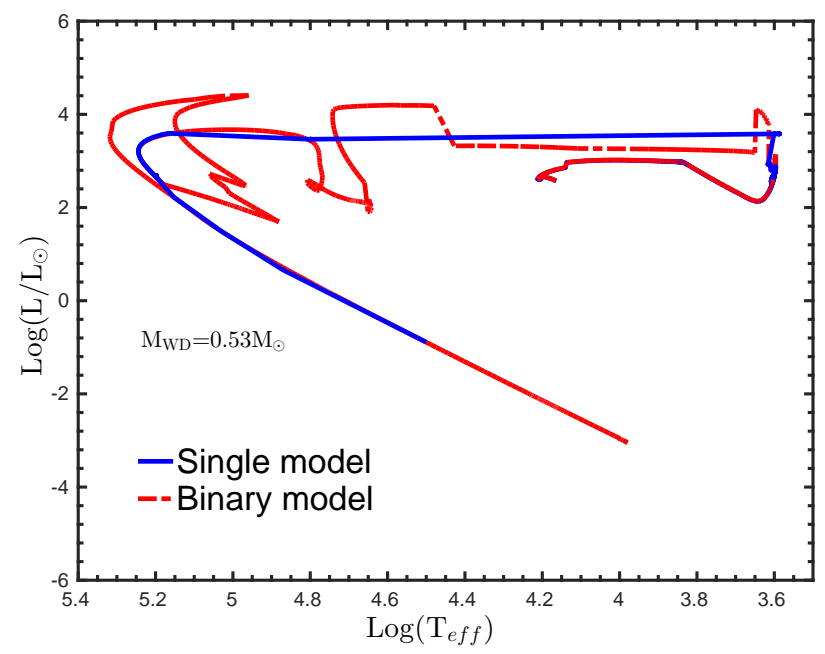

Figure 3. Comparison of the evolution using the single and binary modes. Both models begin at the same position, and mass stripping is modeled as to occur at the same evolutionary stage in both models (specifically we stripping begins once $\mathrm{L}_{\text {nuc }} / \mathrm{L}_{\text {ZAMS }}>0.901$ in this case). Though the early evolution is naturally different, the two evolutionary modes converge once the effective mass loss used effectively mimic the binary evolution. The excellent correspondence between the models supports our use of single mode models for the cases where binary evolution mode did not numerically converge.

3.6-3.9 hybrids to form in a Hubble time. The birthrate of hybrids as a function of the (delay) time between the zeroage (ZAMS) main-sequence and the formation of the hybrid is shown in Fig. 7 (solid black line). In the first 2 Gyrs, the birthrate is about $10^{-12}$ per yr per solar mass of created stars, and it then decreases to a few $\times 10^{-14} \mathrm{yr}^{-1} \mathrm{M}_{\odot}^{-1}$. To put the birthrate of hybrids in perspective, we also show in Fig. 7 the birthrate distribution of white dwarfs of all flavours that are formed in interacting binaries. One Gyr after star
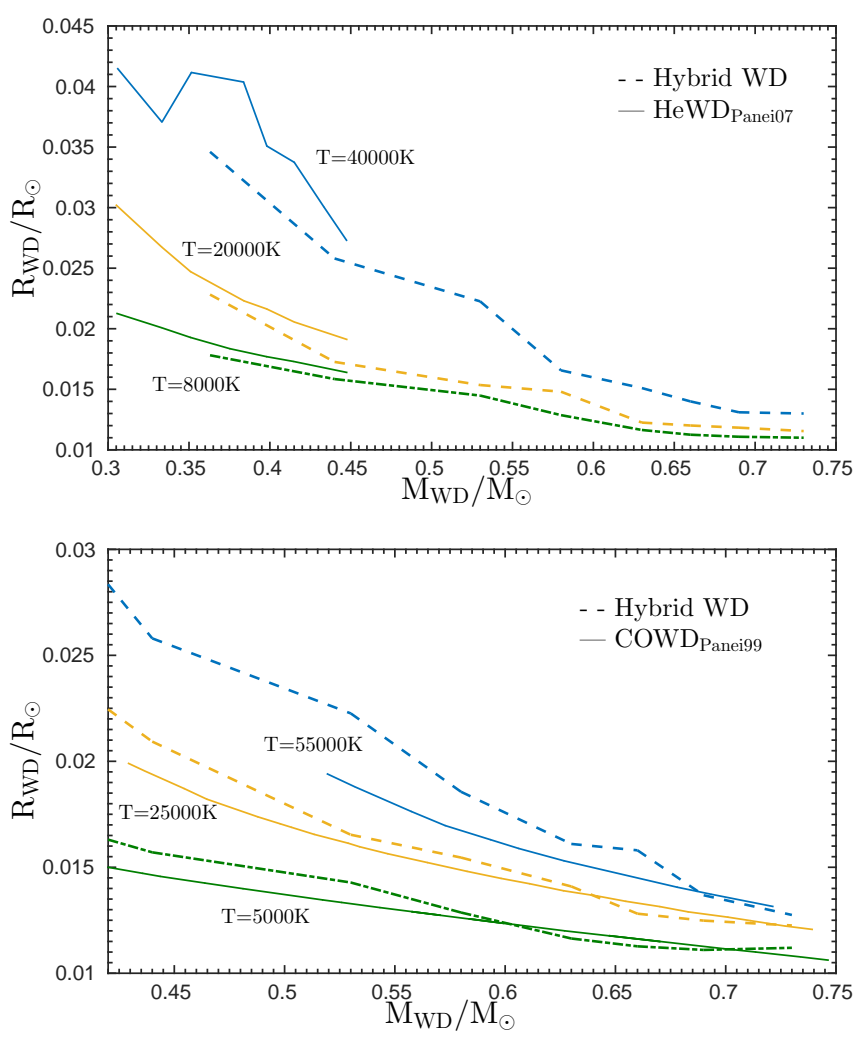

Figure 4. The mass radius relation for hybrid HeCO WDs. The mass radius relation is shown for three temperatures for each regime (comparison with $\mathrm{He}$ WDs on top and with CO WDs in bottom figure). As expected the radii of Hybrid WDs falls in an intermediate regime between purely He WDs purely CO WDs (e.g. compare with Panei et al. 2000, 2007), besides for the most massive hybrids where the He fraction is small and the radii are comparable with that of CO WDs with a small He envelope (taken from Panei et al. 2000, 2007). 

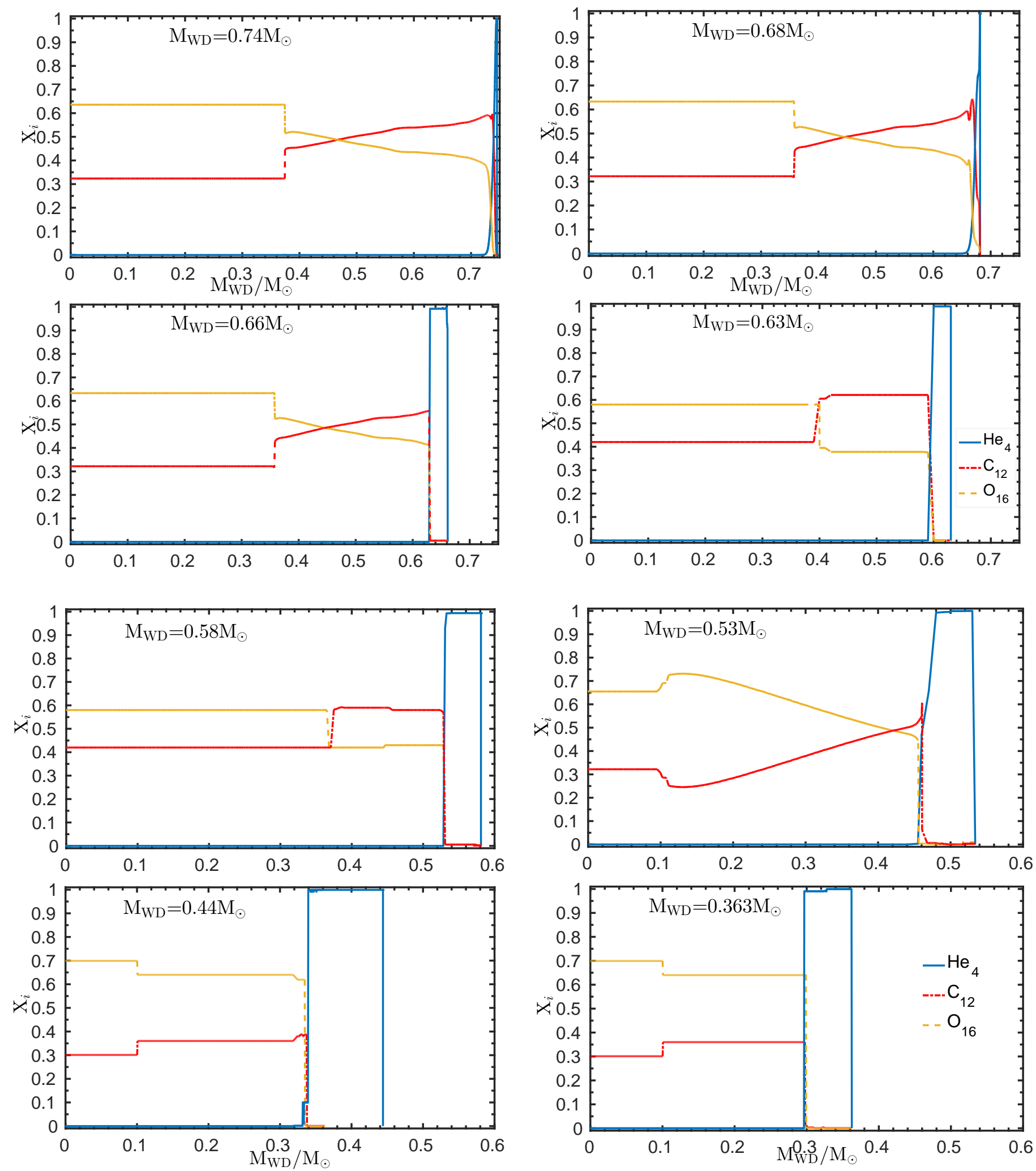

Figure 5. The composition structure of hybrid HeCO WDs for all the calculated models (see Table 1). 

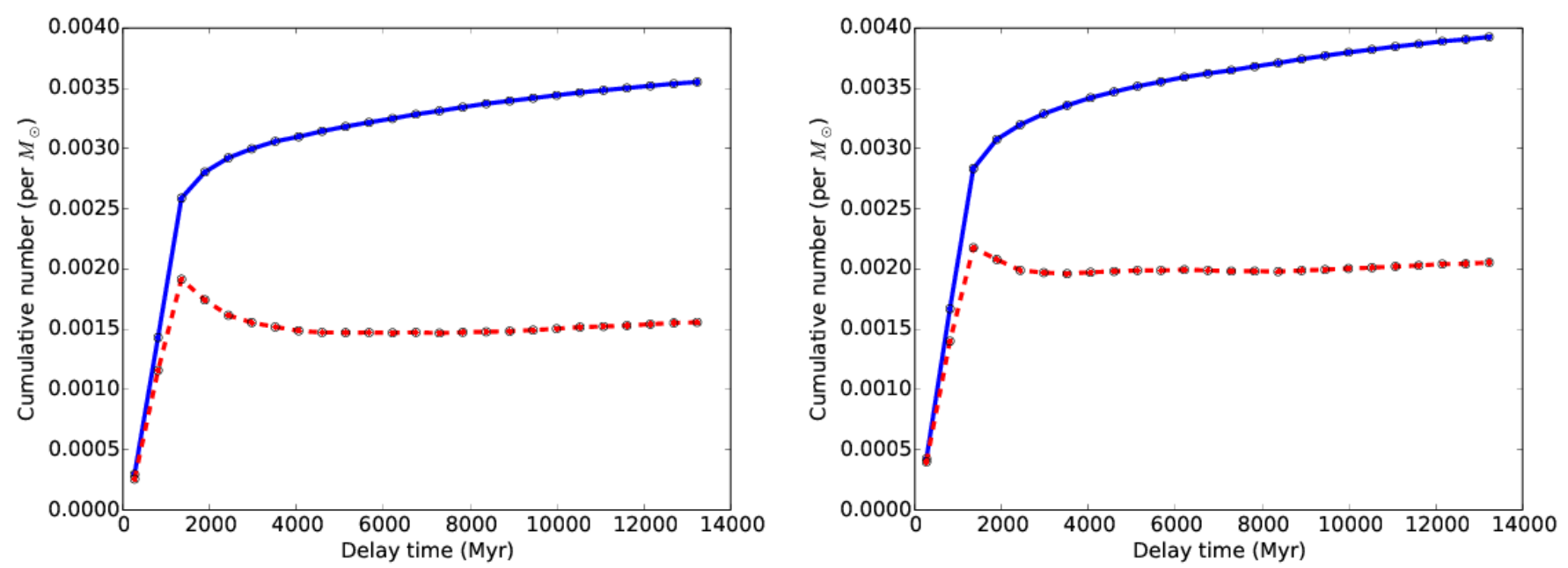

Figure 6. The total cumulative number (per solar mass) of hybrid-WD formed as a function of time. On the left model $\alpha \alpha$ for the CE-phase is shown, on the right model $\gamma \alpha$. Upper (blue) lines show the time integrated birth-rate of hybrid-WDs, i.e. the cumulative number of formed hybrid-WDs as a function of the delay time. Lower (red) line show the total cumulative number of existing hybrids as a function of the delay time, i.e. after accounting for the actual lifetime - subtracting the number of hybrids destroyed (typically through mergers with their companion) from the total number of formed hybrids.
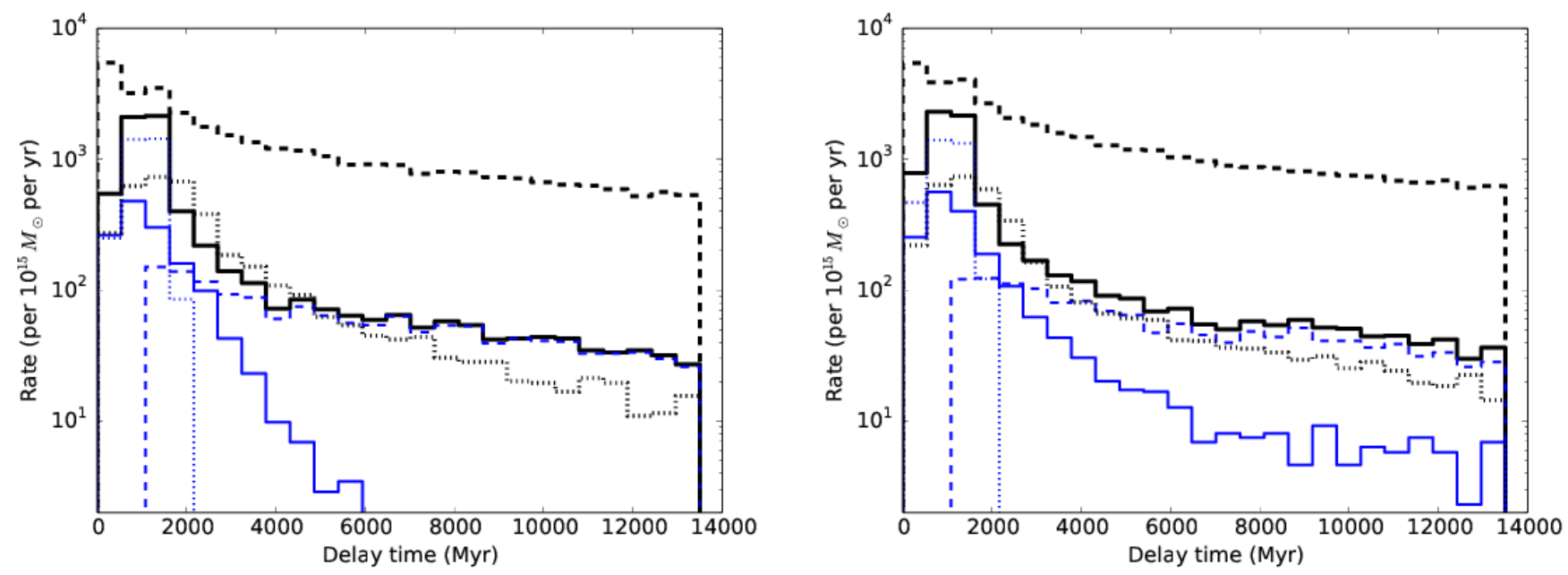

Figure 7. The delay time distribution for the formation of hybrid WDs. Model $\alpha \alpha$ and model $\gamma \alpha$ for the CE-phase are shown on the left and right, respectively. Dashed (black) lines depict all type of WDs in interacting binaries, solid (black) lines depict only hybrid-WDs. Dotted (black) lines show the merger rate of systems with a hybrid (i.e. their destruction rate), dotted (thin blue) lines correspond to the main non-degenerate channel (from more massive stars), while dashed (thin blue) lines depict all hybrids from the degenerate channel (originating from low-mass stars); solid (thin blue) show the cases where the secondary is a hybrid, and the primary is a WD.

formation, roughly $50-60 \%$ of newly born WDs in compact binaries are hybrids. At later time, roughly $6 \%$ of the formedhybrids survive as a hybrid-WDs, since a significant fraction of these binaries merge and are reduced from the overall population.

\subsubsection{Formation channels}

Most hybrids are formed from intermediate mass stars in the first channel at a time-integrated birthrate of $2.7-3.0 \times 10^{-3}$ per $\mathrm{M}_{\odot}$ of created stars. The masses of the hybrids span a large range, from a lower limit of $\sim 0.32 \mathrm{M}_{\odot}$ to the up- per limit set to $0.63 \mathrm{M}_{\odot}$. The birthrate in this channel is therefore sensitive to our assumption about the minimum He fraction of a hybrid. If we are more (less) conservative with our assumption of what constitutes a hybrid WD, and include hybrids with He mass fractions only down to $10 \%$ $(1 \%)$, the time-integrated birthrate from the first channel decreases (increases) to $2.0-2.3 \times 10^{-3}\left(3.2-3.4 \times 10^{-3}\right)$ per $\mathrm{M}_{\odot}$ of created stars. The full range of masses is reached by hybrid progenitors that fill their Roche lobe on, or before the RGB; if the progenitor loses its hydrogen envelope on the $\mathrm{AGB}$, the minimum hybrid mass is $\sim 0.52 M_{\odot}$. The progenitors of the hybrids in this channel have typical ZAMS masses 
of $2-4 \mathrm{M}_{\odot}$, and their formation times are short, i.e. typically several hundred Myrs. This evolutionary channel therefore gives rise to low-mass WD with masses comparable to those of CO WDs typically formed only after Gyrs of evolution of single stars. The formation of hybrid is nevertheless not limited to this short timescale, but can extend up to several Gyrs for hybrid that form only after the companion already has become a WD (Fig. 7).

The time-integrated birthrate of the second evolutionary channel is about $8.3-9.4 \times 10^{-4}$ per $\mathrm{M}_{\odot}$ of created stars. Hybrids from this channel have a mass between $\sim 0.32$ and $0.48 \mathrm{M}_{\odot}$. However, as discussed earlier, such WDs are not likely to be bona-fide hybrid WDs and produce only minute fractions of CO. Their formation times are typically long, ranging up to several Gyrs (Fig. 7). Initially, the ZAMS progenitors of the hybrids are $1-2 \mathrm{M}_{\odot}$ stars predominantly in a (circularized) orbit of about $200-500 \mathrm{R}_{\odot}$. Typically the companion is hydrogen-rich when the hybrid WD is formed. As can be seen, their overall contribution is small compared to the first formation channel from more massive progenitors, besides late times of a few Gyrs.

\subsubsection{Evolution and mergers of hybrid-WDs}

In roughly half $(48-56 \%)$ of the cases, the stellar components of the hybrid-WD binaries will eventually merge with one another through their late evolution. In $25-37 \%$ of these mergers the hybrids merge with hydrogen-rich stellar companions, and in the other systems the hybrids merge with WD companions. Given their large fractions among binary WDs, this suggest that a large fraction of all WD mergers involve a hybrid WD. The merger rate of hybrid white dwarfs is shown in Fig. 7 as a function of the merger time (since ZAMS). After $\sim 4 \mathrm{Gyr}$, a steady state is reached between the formation and destruction rates of hybrid WDs, such that about there is about $1.5-2$ hybrid per $1000 \mathrm{M}_{\odot}$ of created stars (Fig. 6). This later-formed WDs, likely contain only a small fraction of $\mathrm{CO}$, and are effectively He WDs, rather than bona-fide hybrids.

Another likely outcome is that the binary does not change significantly after the formation of the hybrid, e.g. in cases where the secondary is a low-mass MS star that does not evolve off the MS in a Hubble time. This happens for about $\sim 20-26 \%$ of the hybrids. In $14-31 \%$ the binary experiences one or more phases of mass transfer initiated by the secondary star and forms a double white dwarf system. In about $2.0-3.9 \%$ of systems a cataclysmic variable is formed.

\section{DISCUSSION AND SUMMARY}

In this work we have systematically studied the formation and evolution of hybrid HeCO WDs in binary systems. We studied a wide range of initial conditions, and explored the distribution of the hybrid-WDs properties and formation times using binary population synthesis models. Our findings suggest that hybrid HeCO WDs can form robustly, and give rise to a significant fraction of all WD binaries with $50-60 \%$ of all young, < 2Gyr WD being hybrids, (but they become rare among older populations) In particular, a large fraction of all VLM WDs, especially young ones, typically considered to be He WDs could in fact be hybrids. Moreover, the mass range of hybrid WDs can extend up above to $\sim 0.62 \mathrm{M}_{\odot}$, i.e. into the regime typically considered only for CO WDs. Therefore, observationally hybrid WDs could be misidentified as CO DB WDs, which could affect massradius interpretations of observations. Similarly, misidentification could also affect WD chronology estimates, given the difference in the cooling-sequences for hybrids. The hybrid WDs are composed of significant He abundance, with $\mathrm{He}$ mass fractions in the range $2-25 \%$; they reside in an intermediate mass-radius range between purely He and purely CO WDs. The latter issue, which is not included in population synthesis studies may slightly affect the later evolution and interaction of WD-WD binaries, which can strongly depend on the WD radii. We postpone further exploration of this issue for future studies. We note that the different structure and composition of hybrid WDs compared with same mass purely-CO/He WDs could, in principle be probed using asteroseismology (e.g. Winget \& Kepler 2008).

Given their prevalence among WD binaries, hybrid WDs may later further interact with their binary companions. In particular their role in merger of double degenerate systems (WD-WD, WD-NS, WD-BH) could be of particular interest. Such hybrids and their mergers could potentially give rise to explosive thermonuclear events with distinct properties due to the important role of the He in catalyzing more effective thermonuclear reactions and detonations (e.g. in the context of Sub-Chandrasekhar SN explosions Branch \& Nomoto 1986; Woosley et al. 1986; Livne \& Glasner 1990; Iben et al. 1997; Bildsten et al. 2007; Perets et al. 2010; Waldman et al. 2011; WD mergers Pakmor et al. (2013); NS-WD mergers; e.g. Margalit \& Metzger 2016; and WD collisions; e.g. Papish \& Perets 2016). Further studies of these channels using our newly developed detailed hybrid WD models will be further explored in forthcoming publications.

\section{ACKNOWLEDGMENTS}

We thank Bill Wolf, Rob Farmer, Ylva Gotberg and Erez Michaely for stimulating discussions. We acknowledge support from the Israel Science Foundation I-CORE grant 1829/12. ST acknowledges support from the Netherlands Research Council NWO (grant VENI [\#639.041.645]).

\section{REFERENCES}

Abt H. A., 1983, ARA\&A, 21, 343

Althaus L. G., Córsico A. H., Gautschy A., Han Z., Serenelli A. M., Panei J. A., 2004, MNRAS, 347, 125

Bildsten L., Shen K. J., Weinberg N. N., Nelemans G., 2007, ApJ, 662, L95

Branch D., Nomoto K., 1986, A\&A, 164, L13

De Rosa R. J., et al., 2014, MNRAS, 437, 1216

Duchêne G., Kraus A., 2013, ARA\&A, 51, 269

Farmer R., Fields C. E., Petermann I., Dessart L., Cantiello M., Paxton B., Timmes F. X., 2016, ApJS, 227, 22

Han Z., Podsiadlowski P., Maxted P. F. L., Marsh T. R., Ivanova N., 2002, MNRAS, 336, 449

Heggie D. C., 1975, MNRAS, 173, 729

Hurley J. R., Pols O. R., Tout C. A., 2000, MNRAS, 315, 543

Iben Jr. I., Tutukov A. V., 1985, ApJS, 58, 661 
Iben Jr. I., Nomoto K., Tornambe A., Tutukov A. V., 1987, ApJ, 317,717

Iben I. J., Tutukov A. V., Yungelson L. R., 1997, ApJ, 475, 291

Istrate A. G., Marchant P., Tauris T. M., Langer N., Stancliffe R. J., Grassitelli L., 2016, A\&A, 595, A35

Ivanova N., et al., 2013, A\&ARv, 21, 59

Kroupa P., Tout C. A., Gilmore G., 1993, MNRAS, 262, 545

Livne E., Glasner A. S., 1990, ApJ, 361, 244

Margalit B., Metzger B. D., 2016, MNRAS, 461, 1154

Nelemans G., 2010, Ap\&SS, 329, 25

Nelemans G., Verbunt F., Yungelson L. R., Portegies Zwart S. F., 2000, A\&A, 360, 1011

Nelemans G., Portegies Zwart S. F., Verbunt F., Yungelson L. R., 2001, A\&A, 368, 939

Pakmor R., Kromer M., Taubenberger S., Springel V., 2013, ApJ, 770, L8

Panei J. A., Althaus L. G., Benvenuto O. G., 2000, A\&A, 353, 970

Panei J. A., Althaus L. G., Chen X., Han Z., 2007, MNRAS, 382, 779

Papish O., Perets H. B., 2016, ApJ, 822, 19

Paxton B., Bildsten L., Dotter A., Herwig F., Lesaffre P., Timmes F., 2011, ApJS, 192, 3

Paxton B., et al., 2015, ApJS, 220, 15

Perets H. B., et al., 2010, Nature, 465, 322

Portegies Zwart S. F., Verbunt F., 1996, A\&A, 309, 179

Prada Moroni P. G., Straniero O., 2009, A\&A, 507, 1575

Raghavan D., et al., 2010, ApJS, 190, 1

Rebassa-Mansergas A., Nebot Gómez-Morán A., Schreiber M. R., Girven J., Gänsicke B. T., 2011, MNRAS, 413, 1121

Toonen S., Nelemans G., 2013, A\&A, 557, A87

Toonen S., Nelemans G., Portegies Zwart S., 2012, A\&A, 546, A70

Toonen S., Claeys J. S. W., Mennekens N., Ruiter A. J., 2014, A\&A, 562, A14

Toonen S., Hollands M., Gänsicke B. T., Boekholt T., 2017, A\&A, 602, A16

Tutukov A. V., Yungelson L. R., 1992, Soviet Ast., 36, 266

Waldman R., Sauer D., Livne E., Perets H., Glasner A., Mazzali P., Truran J. W., Gal-Yam A., 2011, ApJ, 738, 21

Winget D. E., Kepler S. O., 2008, ARA\&A, 46, 157

Woosley S. E., Taam R. E., Weaver T. A., 1986, ApJ, 301, 601

Zhang X., Hall P. D., Jeffery C. S., Bi S., 2018, MNRAS, 474, 427

This paper has been typeset from a TEX/LATEX file prepared by the author. 